\title{
Structure, Activity, and Deactivation Mechanisms in Double Metal Cyanide Catalysts for the Production of Polyols
}

\author{
Neyvis Almora-Barrios, ${ }^{[a]}$ Sergey Pogodin, ${ }^{[a]}$ Luca Bellarosa $_{,}^{[a]}$ Max García-Melchor, ${ }^{[a]}$ \\ Guillem Revilla-López, ${ }^{[a]}$ Miquel García-Ratés, ${ }^{[a]}$ Ana Belén Vázquez-García, ${ }^{[b]}$ \\ Pedro Hernández-Ariznavarreta, ${ }^{[b]}$ and Núria López ${ }^{{ }^{[a]}}$
}

\begin{abstract}
Polyether polyols are used widely in the plastic and coating industries in the form of polyurethanes. The polymerization of epoxides can be catalyzed by double metal cyanides (DMCs), $\mathrm{Zn}_{3}\left[\mathrm{Co}(\mathrm{CN})_{6}\right]_{2}$. These catalysts were first reported in the $1960 \mathrm{~s}$ by General Tire Inc. and provide products with excellent technical features, which are better than those that result from traditional alkaline polymerization as side reactions are alleviated. However, DMC-catalyzed polymerization is not free of drawbacks as high-molecular-weight side products (1-3 wt \%) can form in the propylene process. These tails are detrimental to
\end{abstract}

the subsequent use of these polymers, in particular to foam stability. Despite the wide industrial interest in DMCs, there are only a few experimental studies and a complete lack of theoretical research of their structure, activity, and performance. The present work is thus the first attempt to describe the nature of the active site, the main polymerization mechanism, and two potential origins for the high-weight tails from a theoretical standpoint by analyzing three crucial steps in the polymerization process.

\section{Introduction}

The polymerization of C2 and C3 epoxides to polyols has been performed traditionally by adding a base, typically $\mathrm{KOH}$, but side reactions with isomerizations to allyl alcohol, ${ }^{[1]}$ detrimental to production, were observed. In the 1960s, General Tire Inc. ${ }^{[2]}$ described a new class of double metal cyanide (DMC) compounds, $\mathrm{Zn}_{3}\left[\mathrm{Co}(\mathrm{CN})_{6}\right]_{2}$, which are active catalysts in this process. The crystal structure of the parent compound was reported as $\mathrm{Zn}_{3}\left[\mathrm{Co}(\mathrm{CN})_{6}\right]_{2} \cdot 12 \mathrm{H}_{2} \mathrm{O}^{[3]}$ and the catalyst was claimed to be heterogeneous in nature. ${ }^{[4]}$ Thereafter, these materials have been optimized by different companies and have become the catalyst of choice for the production of polyether polyols. DMCs are able to polymerize epoxides, namely, propylene oxide, to lead to high-quality polymers that present the following characteristics: 1) the weight distribution is narrower than that with base-induced polymerization, 2) the polymers exhibit a low amount of unsaturations, and 3) the process prevents the side reactions that are typical in alkaline polymerization. The typical characteristics of the polyols made with DMC cata-

[a] Dr. N. Almora-Barrios, Dr. S. Pogodin, Dr. L. Bellarosa,

Dr. M. García-Melchor, Dr. G. Revilla-López, Dr. M. García-Ratés,

Prof. N. López

Institute of Chemical Research of Catalonia, ICIQ, Av.

Paisos Catalans 16, 43007 Tarragona (Spain)

E-mail:nlopez@iciq.es

[b] A. B. Vázquez-García, P. Hernández-Ariznavarreta REPSOL Technology Center Ctra. Extremadura Km. 18, 28935 Móstoles (Spain)

Supporting information for this article is available on the WWW under http://dx.doi.org/10.1002/cctc.201402907. lysts are very high molecular weights and average hydroxyl functionalities of 500-50000 Da. ${ }^{[2,3]}$ As DMCs are very active, the amount of catalyst needed is rather small.

The degree of crystallinity plays a fundamental role in the activity of the catalyst. The higher the degree of amorphicity, the most active the catalyst. ${ }^{[5]}$ To achieve a significant amount of amorphization, the synthesis of the catalysts besides $\mathrm{Zn}$ and Co salts included complexing agents (CAs) to induce amorphicity during growth. ${ }^{[6]} \mathrm{CA}$ modifiers include ethanol, isopropyl alcohol, $n$-butyl alcohol, sec-butyl alcohol, tert-butyl alcohol, isobutyl alcohol, and their mixtures, ${ }^{[5]}$ but also ketones, esters amides, ureas, ${ }^{[2 a, 7]}$ ionic liquids, ${ }^{[8]}$ and glyme (dimethoxyethane $)^{[9]}$ have been proposed. The reaction shows an induction period that was suggested to be caused by some degree of ligand replacement and structural changes. ${ }^{[9]}$

Since the initial formulation, several synthetic processes have been proposed ${ }^{[10]}$ for instance, the reaction of an aqueous solution of $\mathrm{ZnCl}_{2}$ and other secondary metal salts such as $\mathrm{K}_{3} \mathrm{Fe}(\mathrm{CN})_{6}$ with $\mathrm{CA}$. Zhang et al. ${ }^{[6]}$ investigated the role of $\mathrm{ZnCl}_{2}$ and different $C A s$ in the polymerization of propylene oxide and described a potential mechanism for the reaction. They found that excess $\mathrm{ZnCl}_{2}$ was ultimately responsible for the polymerization. However, free $\mathrm{ZnCl}_{2}$ was not active by itself. ${ }^{[6]}$ Therefore, in the active catalyst, $\mathrm{ZnCl}_{2}$ was suggested to be incorporated to the lattice in the form of $\mathrm{CN}-\mathrm{Zn}-\mathrm{Cl}$ terminations, which were identified as dormant active sites. An indepth characterization was presented by Chen and Chen, ${ }^{[11]}$ which included tert-butyl alcohol and a co-complexing agent polyether with electron spectroscopy for chemical analysis 
(ESCA), IR spectroscopy, XRD, SEM, and X-ray photoelectron spectroscopy (XPS) experiments performed to characterize them. From these experiments the active site was identified as $\mathrm{Zn}^{2+} \cdot{ }^{[10]}$

Typically, the polymerization is conducted in a conventional batch reactor in which the catalyst and initiator are introduced. Then the reactor is set to the desired temperature (usually between 70 and $150^{\circ} \mathrm{C}$ ) and a portion of the epoxide is added. The sign that the catalyst has been activated is a decrease in pressure. At this point, the epoxide is fed to maintain the internal pressure from 0.7 to 4 bar. ${ }^{[8,12]}$ The activity and induction period with alkoxy alcohols was studied, ${ }^{[13]}$ which showed a sharp decrease in the crystallinity from the stable $\mathrm{Zn}_{3}\left[\mathrm{Co}(\mathrm{CN})_{6}\right]_{2} \cdot 12 \mathrm{H}_{2} \mathrm{O}$ if CA was included. The type of $\mathrm{CA}$ is a crucial factor in the polymerization, which includes induction periods and polymerization rate. Low-polarity CAs were proposed as the best candidates for commercialization. Kim et al. ${ }^{[14]}$ identified that the type and amount of oxygen in the first coordination sphere of $\mathrm{Zn}$ seemed to play an important role in the polymerization as the $\mathrm{Zn}$ atoms are the true active sites. ${ }^{[15]}$ They also identified a fast exchange between dormant and active sites and a reaction mechanism (Scheme 1). Moreover, they illustrated how the coordination of different CAs has a big effect on the activity and initiation periods. ${ }^{[14]}$ Finally, a mechanistic study that included a mathematical model for

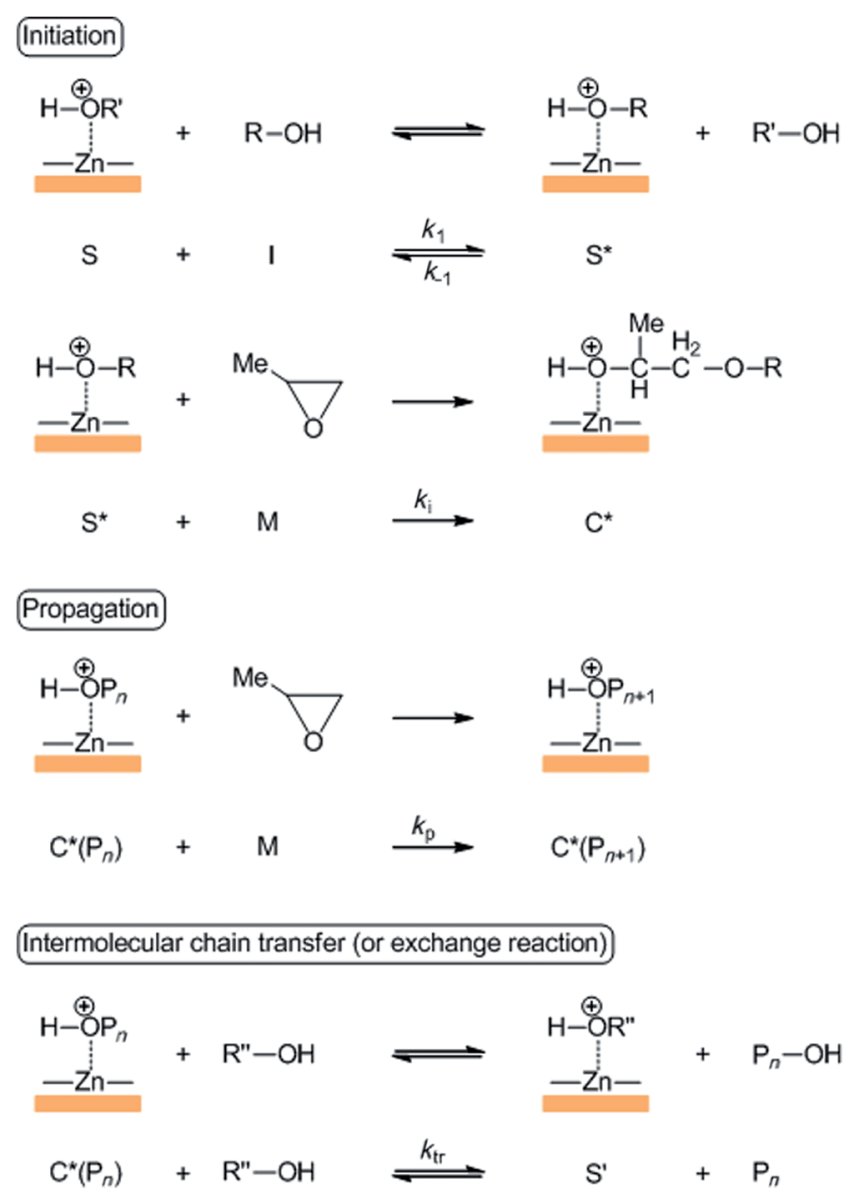

Scheme 1. Reaction mechanism adapted from Ref. [14]. the reaction network was proposed in Ref. [16] for the polymerization process.

Similar DMC catalysts have been suggested for the copolymerization of epoxides with $\mathrm{CO}_{2}$, which expands the catalytic scope of DMC catalysts. For instance, sol-gel synthesis was also applied in the synthetic process of DMC and then tested to copolymerize styrene oxide and $\mathrm{CO}_{2} \cdot{ }^{[17]}$ In this case, a mechanism was put forward and the active system was identified as a semicrystalline $\mathrm{Zn}_{3}[\mathrm{Co}(\mathrm{CN})]_{6}$ phase with excess $\mathrm{Zn}$. CAs were also investigated in semibatch copolymerizations of cyclohexene oxide and $\mathrm{CO}_{2}{ }^{\left[{ }^{8,18]}\right.}$ In all cases, the choice of $\mathrm{CA}$ was identified as the most important single factor to activate $\mathrm{CO}_{2}$ incorporation to the polymer. In addition, the synthesis of nanosized DMC catalysts was performed to achieve synthesis control, ${ }^{[18,19]}$ and the obtained materials were tested for hydrogen storage. ${ }^{[20]}$

The intrinsic properties of DMC catalysts are not understood at the atomic level, mainly because of the difficulties encountered if computational methods are applied to the study of these catalysts. Perhaps the most important hurdle is that related to the intrinsic nature of the catalyst. ${ }^{[21]}$ It is well known that if the catalyst is prepared as a single-crystalline phase, the DMC is poorly active and CAs have to activate the catalyst. If a different $C A$ is used, the degree of crystallinity of the material is reduced, which indicates that the amorphous phase is the truly active phase. In addition to these structural problems, the reaction has not been well characterized yet, and the nature of the active site still remains under debate. Herein, we present the first theoretical study of the polymerization mechanism, as well as the role of different $\mathrm{CAs}(\mathrm{Cl}, \mathrm{OH}, \mathrm{OEt}, \mathrm{OtBu})$. Furthermore, we provide a qualitative description of the reaction network and pay special attention to the first reaction steps and the origin of high-weight polymers with the aim to provide a molecular understanding of this very complex process.

\section{Results and Discussion}

\section{Crystalline structures for prototypical DMC catalysts}

Amorphous materials retain the first coordination sphere and reduce the degree of ordering in the second coordination sphere. ${ }^{[22]}$ Thus, theoretical simulations applied to the crystalline structure of DMC can help to identify the most common coordination patterns in the amorphous structure and also some of the medium-range structures that can provide valuable information on the active site, reaction mechanism, and deactivation routes. Therefore, the models presented here allow us to clarify the active site patterns needed for the reaction to occur. The DMC structure presents a hexagonal unit cell, ${ }^{[23]}$ in which the octahedral Co unit acts as a spacer for the tetrahedral Zn polygons (Figure 1). The loss of secondary order would only mean that the octahedra and tetrahedra would not be as ordered as pictured here but they would still have the same nature.

In our case, we have taken the parent compound in the native DMC structure to reoptimize the cells. A full replacement of the $\mathrm{Cl}$ anions in the lattice by the $\mathrm{CAs} \mathrm{OH}, \mathrm{OEt}$, and 


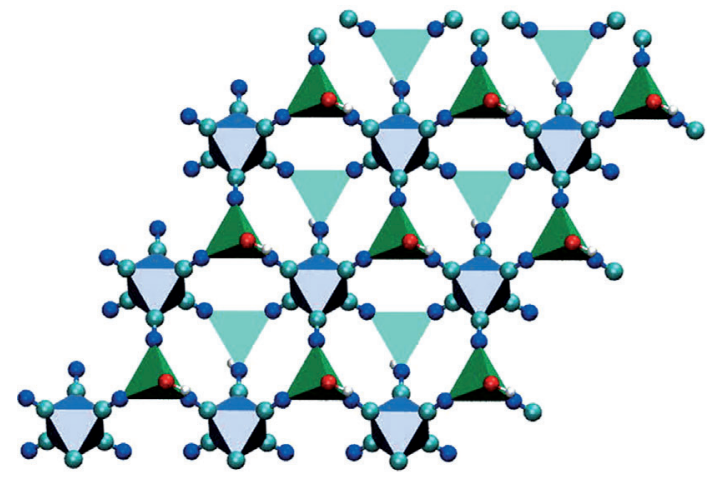

Figure 1. DMC crystal structure. Color code: tetrahedral Zn sites (green), octahedral Co centers (cyan), C (cyan), N(blue), O (red) and H (white) atoms. The $\mathrm{CA}$ in the present case is represented by the terminal red $\mathrm{O}$ in the $\mathrm{OH}$ groups.

$\mathrm{OtBu}$ were examined. Upon replacement, all the bulk cells were reoptimized (Table 1). The structure is kept with some minor changes to adapt the small $\mathrm{OH}$ or the bulky OtBu cen-

\begin{tabular}{|c|c|c|c|c|c|}
\hline$C A$ & $A$ & $b$ & $c$ & $Z n-Z n$ & $Z n-Z n$ \\
\hline $\mathrm{Cl}$ & 7.524 & 7.526 & 18.295 & 4.381 & 6.515 \\
\hline $\mathrm{OH}$ & 7.530 & 7.534 & 17.111 & 3.754 & 6.525 \\
\hline OEt & 7.530 & 7.561 & 16.967 & 3.721 & 6.502 \\
\hline $\mathrm{OtBu}$ & 7.499 & 7.562 & 17.049 & 3.739 & 6.511 \\
\hline
\end{tabular}

ters, but in all cases the separation between the $\mathrm{Zn}$ tetragonal coordination spheres and the octahedral configurations of the robust $\mathrm{Co}(\mathrm{CN})_{6}$ spacers is maintained. The cell parameters observed upon CA replacement are very similar to those of the parent compound. The main changes occur along the $c$ direction for which even a $5 \%$ change is reported for $C A=O E t$. If replaced, the shortest $Z n-Z n$ contacts are reduced to $3.7 \AA$, from an initial value of $4.4 \AA$, and the second $Z n-Z n$ sphere is maintained at a distance of $6.5 \AA$.

A cut along the lowest index surfaces was performed for all these bulk materials with full substitution. For DMC, both $(001)$ and (100) surfaces show the lowest energies. The $(001)$ surface exposes $\mathrm{Zn}$ atoms at the external part with $\mathrm{Zn}$ atoms in the outermost positions spaced by $\mathrm{Co}(\mathrm{CN})_{6}$ motifs (Figures 2 and 3). These layers are bonded to the next through $\mathrm{Cl}$ atoms. The (100) surface is more open, and the $Z n$ tetrahedral motifs are exposed on the surface, however, they are not spaced by $\mathrm{Co}$ as in $(001)$ but linked by $\mathrm{Cl}$ (or the $\mathrm{CA}$ ). Therefore, the $(001)$ surface can be described as isolated single sites, whereas close $\mathrm{Zn}-\mathrm{Zn}$ contacts appear for the (100) surface.

Surface energy calculations for the two main facets are presented in Figure 2. Our results indicate that the $(001)$ surface, in which $\mathrm{Zn}$ atoms are isolated, is more stable regardless of the CA. The lowest energy required to create a surface corresponds to very bulky capping agents as expected from the steric hindrance. Electronic effects also play a role, and linkers

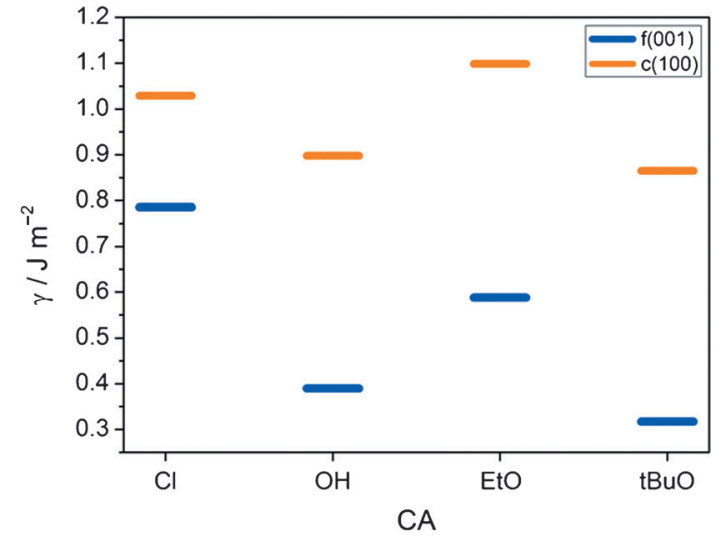

Figure 2. Surface energies, $\gamma$, for the different CAs and the lowest two energy facets shown in Figure 3.

a)
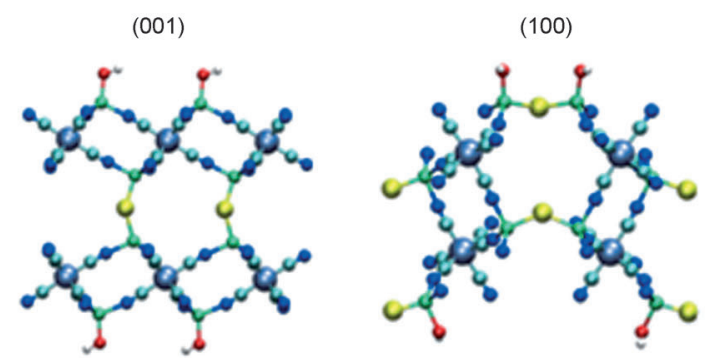

b)
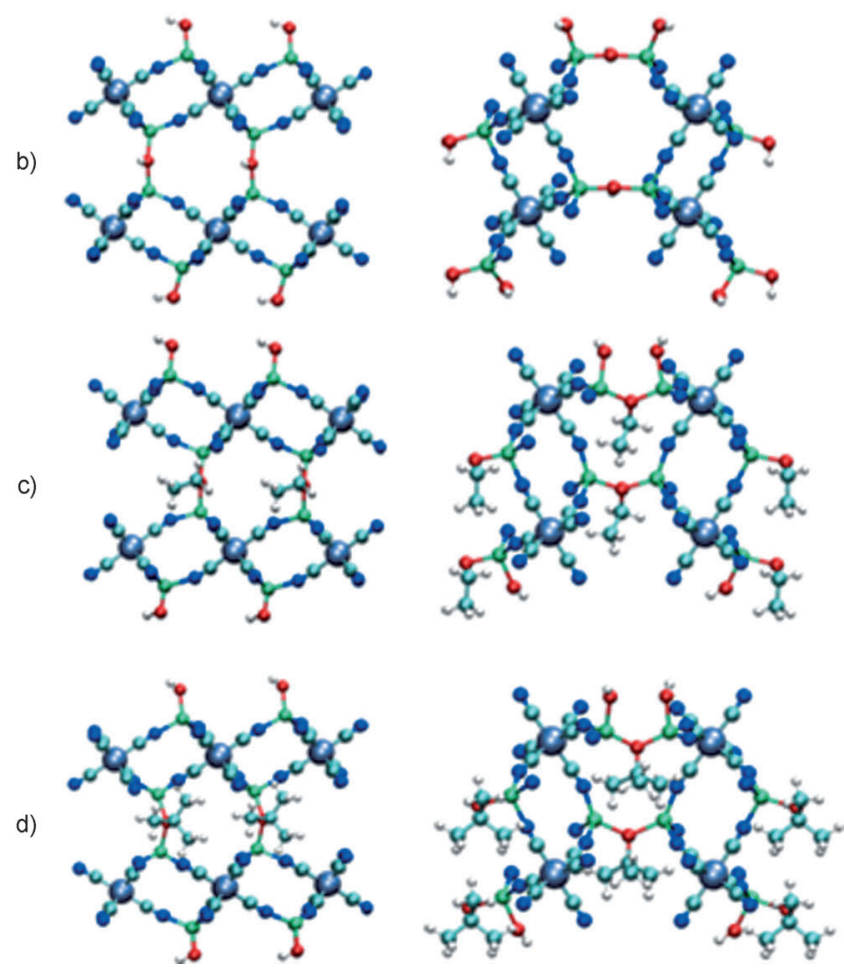

Figure 3. Lowest energy surfaces of DMC with different $\mathrm{CAs}$ : a) $\mathrm{Cl}, \mathrm{b}) \mathrm{OH}$, c) OEt, d) OtBu. Color code: Co centers (large gray/blue) Zn centers (green), $\mathrm{Cl}$ (yellow), C (cyan), N (blue), O (red) and H (white) atoms.

with oxygen fragments stabilize the lowest energy facet significantly. Relative energies, which might affect the ratio between 
isolated and close $\mathrm{Zn}$ centers are also affected significantly by the CA. For instance, the equilibrium nanocrystal shows a more extended (100) facet for EtO as the ratio between the surface energies of $(100)$ and $(001)$ is more favorable.

\section{Reaction profile for DMC catalysts}

Once the most likely structure for the two surfaces was assessed, we next investigated the mechanism for the reactions shown in Scheme 1 for both isolated $Z n$ atoms (001) and the close $\mathrm{Zn}$ surface (100). Ethylene oxide was considered as a polymerization unit. Qualitatively, our path follows the mechanism illustrated by Kim et al., ${ }^{[14]}$ which is summarized in Scheme 1 for the $\mathrm{CA}=\mathrm{OH}$ catalyst. The reaction profiles can be found in Figure S1, in which the data for the reactions summarized in Table 2 and the adsorption energies of incoming epoxides are taken into account.

Table 2. Initiation (12) and propagation (P) steps for the DMC catalyst with $\mathrm{CA}=\mathrm{OH}$ and both $(001)$ and $(100)$ surfaces from coadsorbed reactants. $\Delta E$ is the reaction energy and $E_{\mathrm{a}}$ is the corresponding activation barrier. All energies in $\mathrm{kJ} \mathrm{mol}^{-1}$.

\begin{tabular}{|llrr|}
\hline Reaction & Surface & $\Delta E$ & \multicolumn{1}{c}{$E_{\mathrm{a}}$} \\
\hline $\mathrm{I} 2$ & $(0001)$ & -64 & 148 \\
$\mathrm{I} 2$ & $(100)$ & -145 & 87 \\
$\mathrm{P}$ & $(001)$ & -54 & 156 \\
$\mathrm{P}$ & $(100)$ & -57 & 107 \\
I2-CA & $(100)$ & -43 & 61 \\
\hline
\end{tabular}

Initiation steps:

$$
\begin{aligned}
& \mathrm{HOR}^{\prime}-\mathrm{Zn}+\mathrm{HOR} \leftrightarrow \mathrm{HOR}-\mathrm{Zn}+\mathrm{R}^{\prime} \mathrm{OH} \\
& \mathrm{HOR}-\mathrm{Zn}+\mathrm{EO} \leftrightarrow \mathrm{HOCH}-\mathrm{CH}_{2} \mathrm{OR}-\mathrm{Zn}
\end{aligned}
$$

Propagation steps:

$$
\mathrm{HOCH}-\mathrm{CH}_{2} \mathrm{OR}-\mathrm{Zn}+\mathrm{EO} \leftrightarrow \mathrm{HO}\left(\mathrm{CH}-\mathrm{CH}_{2} \mathrm{O}\right)_{2} \mathrm{R}-\mathrm{Zn}
$$

Termination steps:

$$
\mathrm{HO}\left(\mathrm{CH}-\mathrm{CH}_{2} \mathrm{O}\right)_{n} \mathrm{R}-\mathrm{Zn}+\mathrm{R}^{\prime \prime} \mathrm{OH} \leftrightarrow \mathrm{E}-\mathrm{OH}+\mathrm{Zn}-\mathrm{OR}^{\prime \prime}
$$

In which $\mathrm{HOR}-\mathrm{Zn}$ is the catalyst with the growing polymer, $\mathrm{HOR}$ is the initiator, EO is ethylene oxide, and $\mathrm{R}^{\prime \prime} \mathrm{OH}$ is the terminating agent. As the whole list of reactions is complex and some of them are performed many times with practically no energy variation $(P)$ we will concentrate on a few key steps to understand the activity of the process. The similar character of the propagation steps in chain polymerization is well established. ${ }^{[24]}$

\section{Initial step: $\mathrm{HOR}-\mathrm{Zn}+\mathrm{EO} \leftrightarrow \mathrm{HOCH}-\mathrm{CH}_{2} \mathrm{OR}-\mathrm{Zn}$ (I2)}

The main features of the initiation step are presented in Table 2. Epoxide adsorption is quite weak and dominated by the ability of $\mathrm{Zn}$ atoms to show coordination spheres that range from four to six. ${ }^{[25]}$ Although the adsorption energy of the epoxide is weak, the high pressure of the epoxide in the experiments (4 bar) justifies this adsorption step. Once coordinated, the epoxide is activated and one of the carbon atoms of the epoxide suffers a nucleophilic attack by the terminal $\mathrm{OH}$ group, which results in the formation of a five-membered ring intermediate (Figure 4). This can be opened easily to give
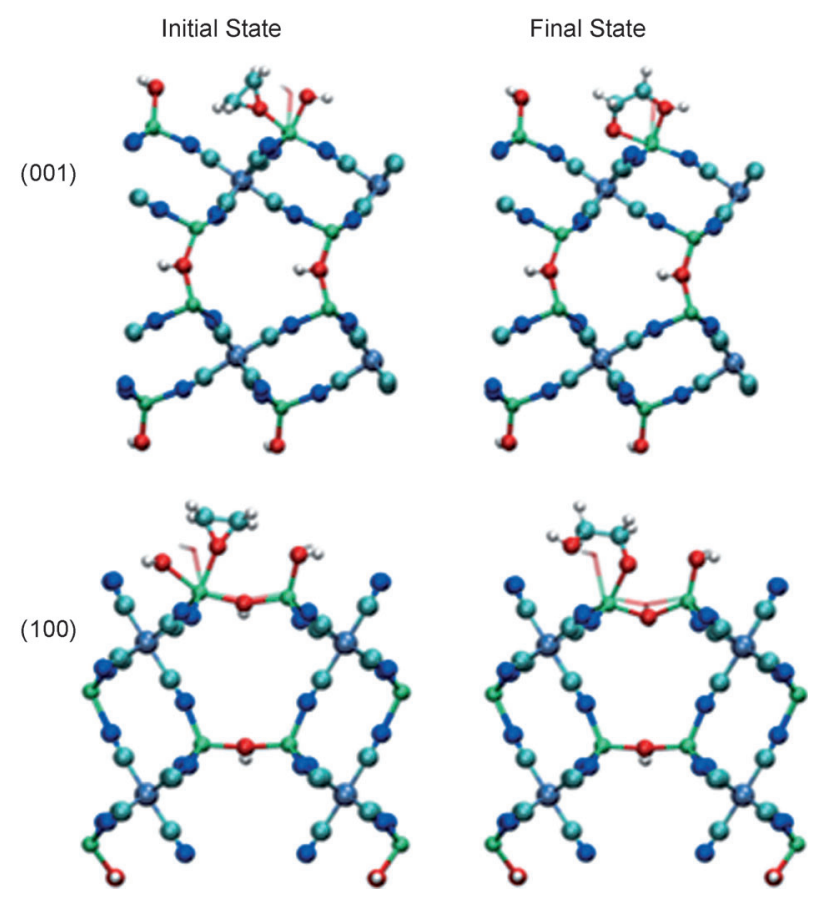

Figure 4. A representation of the initial (coadsorbed reactants) and final state of (I2) reactions on (001) and (100) surfaces of DMC. The color code is the same as in the previous figures.

a four-coordinated $\mathrm{Zn}$ with a growing chain on top, which is the starting point for the propagation step. Reaction on both surfaces is exothermic by approximately $60 \mathrm{~kJ} \mathrm{~mol}^{-1}$ in the (001) surface and close to $150 \mathrm{~kJ} \mathrm{~mol}^{-1}$ for the (100) one. Although the reaction is hindered by a significant barrier of approximately $150 \mathrm{~kJ} \mathrm{~mol}^{-1}$ on the $(001)$ surface, the energy demand is much lower on the open (100) facet (Table 2). Thus, the $(100)$ facet is more active that the corresponding (001) facet.

\section{Propagation: $\mathrm{HOCH}-\mathrm{CH}_{2} \mathrm{OR}-\mathrm{Zn}+\mathrm{EO} \leftrightarrow \mathrm{HO}\left(\mathrm{CH}-\mathrm{CH}_{2} \mathrm{O}\right)_{2} \mathrm{R}-\mathrm{Zn}$ (P)}

The propagation step is repeated with little variation during most of the polymerization process. In the present work, we have concentrated only on the formation of the dimer of the polyol as our interest concerns only the most salient features of this particular step. A summary of the structures and energies is presented in Table 2 and Figure 5. Again, for the propagation step, the adsorption of the epoxide in the $\mathrm{Zn}$ atom in which the chain grows is slightly exothermic by $10-$ $25 \mathrm{~kJ} \mathrm{~mol}^{-1}$. The propagation is exothermic if evaluated in both 


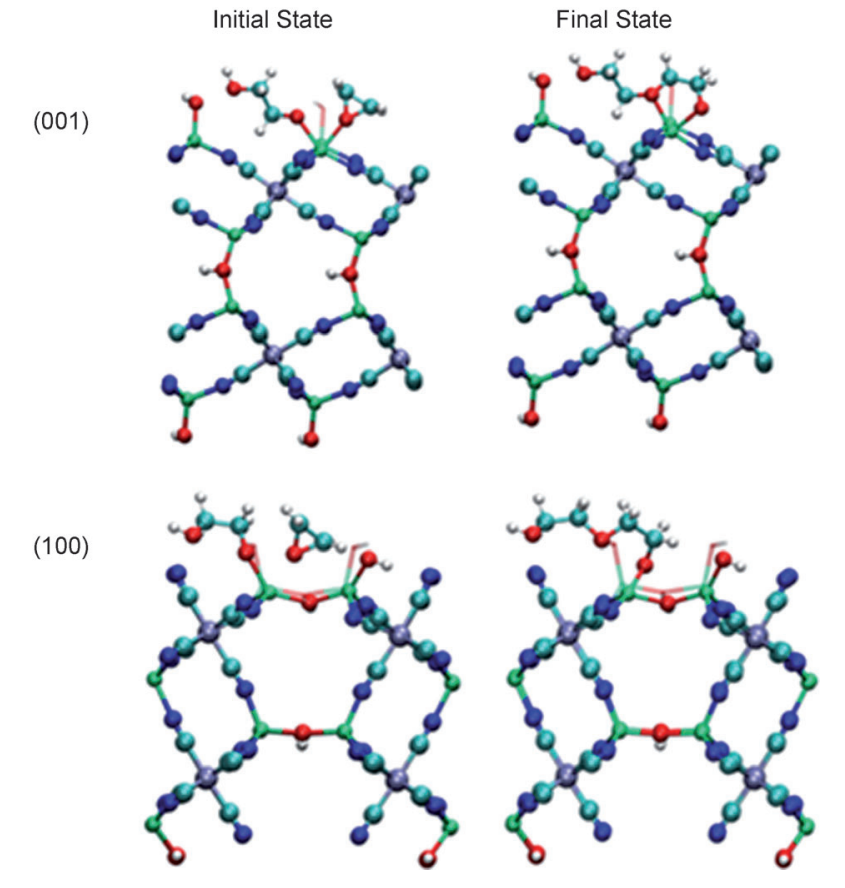

Figure 5. A representation of the initial (coadsorbed reactants) and final state of $(P)$ reactions on $(001)$ and $(100)$ surfaces of DMC. The color code is the same as in previous figures.

the $(001)$ and $(100)$ facets. The corresponding activation energies differ significantly, more than $150 \mathrm{~kJ} \mathrm{~mol}^{-1}$ on (001) and again a much lower energy demand on the (100) facet, an approximately $50 \mathrm{~kJ} \mathrm{~mol}^{-1}$ smaller barrier. As the (P) step is basically repeated in homopolymerization, the step presented here corresponds to a generic $(P)$ profile.

To understand the reason for the lower activation energies found for the (100) surface, we analyzed its electronic structure (Figure 6). The calculated Bader charges show no significant differences for the surface atoms of both facets (Tables S1 and S2). As the activity is controlled by the ability of the reac-

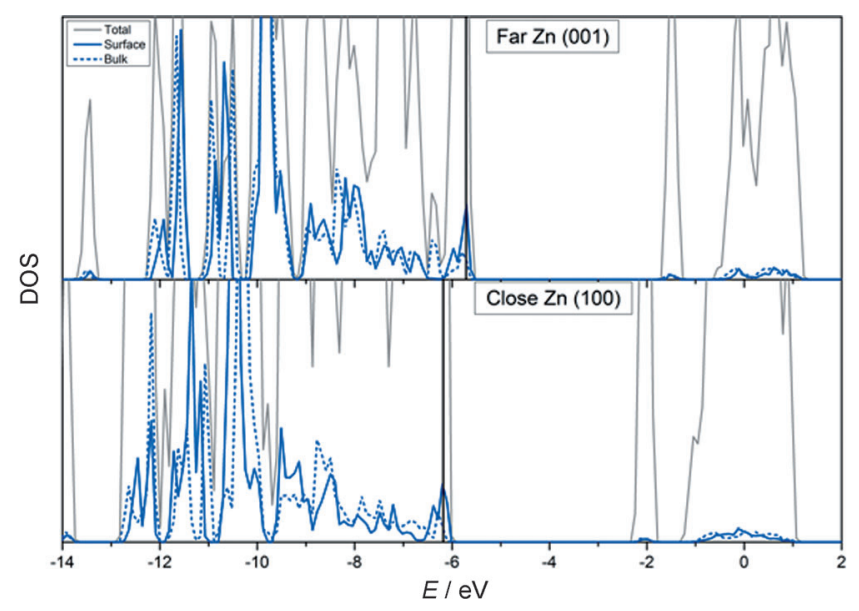

Figure 6. Projected density of states for the two surfaces of the DMC catalyst with $\mathrm{CA}=\mathrm{OH}$. Continuous blue lines correspond to $\mathrm{Zn}$ atoms on the surface, dotted lines correspond to the bulk, and grey ones are the total. The Fermi level is marked by the vertical black line. tants to coordinate to $\mathrm{Zn}$, we analyzed the empty levels that correspond to $\mathrm{Zn}$ as they are responsible for the catalyst Lewis acidic character. These levels are lower in energy, thus electron transfer (or sharing) with the incoming OR groups is very effective. This observation is in close agreement with the experimental determination that $\mathrm{Zn}$ atoms linked to $\mathrm{O}$ atoms are responsible for the high activity observed in the DMC catalysts. ${ }^{[14]}$ Therefore, the $(100)$ surface is more likely to activate the molecules and polymerize them because the number of $O$ groups is larger than that in $(001)$. These results are in agreement with earlier experimental results. ${ }^{[12]}$ Thus CAs that contain oxo groups are well suited to improve the activity of the catalyst. A particular example is $\mathrm{N}$-containing ligands that generate weaker Lewis acids as the crystal field splitting generated is smaller (for instance if $\mathrm{SCN}$ and $\mathrm{OH}^{-}$groups are compared). This activation does not affect further polymerization steps as in all cases the growing polymer is bound to the $\mathrm{Zn}$ active site through an OR group and it is not removed from the active site until it reaches a high weight, up to $50000 \mathrm{Da}$. However, the changes caused by CAs with a higher $O$ content also have their own drawbacks as we will see later.

\section{Routes to deactivation: Origins of the formation of high- weight tails}

A side problem of DMC catalysts in polyether polyol polymerization might be related to the high activity reported. Together with the narrow polymer distribution there is a certain amount of high-weight polymers. These tails prevent the formation of stable foams, one of the major applications of polyols. Highmolecular-weight polymers were identified as $2-3 \%$ of the weight of the total sample. ${ }^{[12]}$ These species have a surfactantlike effect that alters the solubility and produces the phasingout of the polyurethane polymers in the isocyanate-polyol foam synthesis. The tail was more abundant if $\mathrm{Zn}-\mathrm{OH}$ groups were present. ${ }^{[12]}$ The same patent identifies the interaction with a protic acid (such as acetic acid) for some time as able to abate the problem. ${ }^{[12]}$ The acid was found to replace the $\mathrm{Zn}-\mathrm{OH}$ vibrational fingerprints with $\mathrm{Zn}$-acetate ones. In that case, the use of a solvent that was aqueous in character was found to be positive. The topology of site isolation is important in the patents, ${ }^{[26]}$ and the presence of EO in a fraction of the polymer (i.e., a reduction of the amount of $\mathrm{PO}$ ) was also claimed to be positive to avoid the formation of high-weight tails. The nature of the tails, either hydrophobic or hydrophilic, also had a role. ${ }^{[26]}$

The formation of such tails might have two explanations with a covalent (bonding) origin: 1) presence of multiple active sites and 2) the ramification of the polymer by following the paths described in the previous section. Given the nature of the $\mathrm{Zn}$ active sites in the (100) surface, we have identified two potential routes that can alter the behavior described for normal polymer growth. The first is explained by the fact that the surface $\mathrm{Zn}$ atoms in the (100) expose the $\mathrm{OH}$ linker between the $\mathrm{Zn}$ atoms to the surface so they can be easily incorporated into the polymer. To analyze this possibility we have investigated the possibility that reaction 12 takes with the CA 
$(100)$

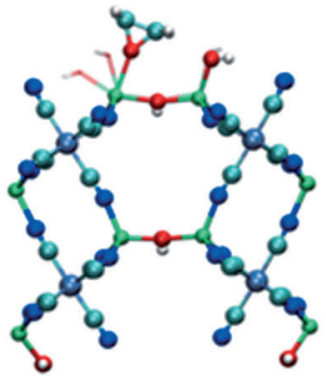

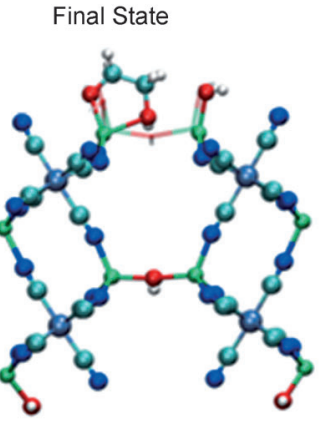

Figure 7. A representation of the initial (coadsorbed reactants) and final state of the alternative $(I 2-C A)$ reaction on $(100)$ surfaces of DMC. The color code is the same as in previous figures.

instead of the initiating agent, and the results are shown in Table 2 and Figure 7.

As seen from the results, the incorporation of the CA to the chain is relatively easy as the process is exothermic and the barrier is low. The computed barrier might be exceedingly low if we consider that the CA employed in the model is the smallest $\mathrm{OH}$ group (compared to typical OEt or OtBu groups). Moreover, this path shows that the $(100)$ surface might be destabilized by the opening of the $\mathrm{Zn}-\mathrm{O}-\mathrm{Zn}$ bridge. Therefore, the growing polymer would have two different positions from which the propagation steps could take place simultaneously. Unfortunately, the identification of the CA incorporated is experimentally impossible because of the high weight of the polymer.

In addition, the incorporation of the $C A$ into the polymer has an extra effect as the neighboring $\mathrm{Zn}$ atoms can be more exposed to the reaction mixture and other polyols can grow in the nascent active $\mathrm{Zn}$. Thus atoms in the bulk of the material can become active centers, which would result in a higher activity. This observation explains the activation step needed for the catalyst to be more active. In principle, this result is positive in terms of production. The second effect corresponds to the interference between different sites and it also affects open surfaces in which the $\mathrm{Zn}$ atoms are separated by a linker such as the (100) surface. Given the distribution of the $\mathrm{Zn}$ atoms on this surface, it is possible to form a polyol with more than one active head. We present the summary of one of these potential paths in Table 2.

From the process described in Table 2, it can be seen that the cross-interaction between two different sites is indeed possible. In this case, the reaction is again exothermic and the stress of the $\mathrm{O}-\mathrm{C}-\mathrm{C}-\mathrm{O}$ chain can be accommodated easily in the final structure. The reaction shows a slightly larger activation barrier than that reported in previous sections. However, under reaction conditions $T=130^{\circ} \mathrm{C}$ and 4 bar, epoxide pressure cannot be discarded. As we have mentioned, the specific concentration of the nonisolated $\mathrm{Zn}$ sites cannot be specified as the $\mathrm{Zn}$ coordination sphere and the $\mathrm{Zn}-\mathrm{O}$ bonds are both very labile. ${ }^{[25]}$ Thus, it is possible that although the $\mathrm{Zn}-\mathrm{Zn}$ atoms are not interconnected through the catalyst lattice but happen to be physically close because of the amorphization of the solid or the polymerization-induced breaking of the materi-

al, this chemical interconnection of the chains leads to multiple heads that polymerize in a single chain.

There is yet an alternative potential origin for the highweight fraction polyol chains related to the physical (noncovalent) interaction between different chains that leads to chain entangling. This process is physical and thus it can only be presented here with qualitative arguments. The polymers grow in the isolated site $\mathrm{Zn}$ centers in the catalyst, which is quite diluted, and in principle they might seem as isolated chains once the catalyst is activated. However, if step $(P)$ is repeated a few times the growing chains that did not interact previously might be not isolated anymore. Indeed, the simultaneous formation of multiple molecular chains on near active sites leads to the formation of a polymer brush with a dense interweaving of the polymer backbones. For those, numerous intersections of the backbone trajectories effectively create topological entanglements of the growing molecules, not easy to break apart once the produced polymers are far from the catalyst. Actually, estimations in the framework of reptation theory by Doi and Edwards $^{[27]}$ suggest that the diffusion coefficient of the center of mass of a polymer chain in a melt of many other chains is proportional to $D_{\mathrm{G}} \propto N_{0}^{-2} \rho^{-2 \alpha}$, in which $N_{0}$ is the length of the polymer chain in terms of Kuhn segments, $\rho$ is the mass concentration of polymer, and $\alpha$ is approximately $0.60 / 0.75$. Although an accurate quantitative characterization of the effect on the discussed polymerization reaction is challenging, the way to avoid it is clear: the larger the distance between the active sites with polymers that are growing simultaneously, the fewer entanglements are formed in the product (Figure 8). This is in agreement with earlier reports. ${ }^{[18]}$
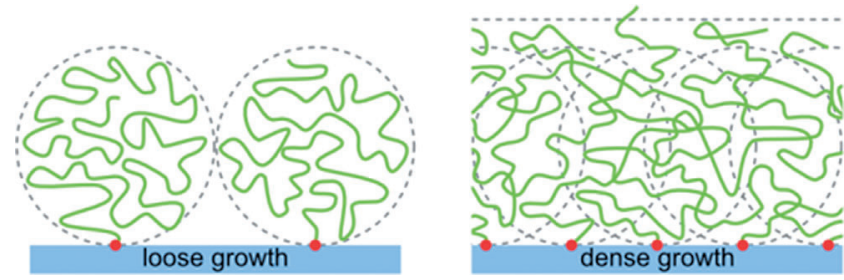

Figure 8. Surface growth of polymer chains at low and high density of active sites.

Moreover, the interaction with different chains will also be affected by the polarity, $\mathrm{pH}$ and ionic force of the media in which the polymer grows. In this case, whether EO or PO is the polymerization monomer plays a fundamental role in the degree of extension of the chain.

\section{Design rules for DMC and modulators to prevent high- weight tails}

The theoretical modeling described here identifies the key factors of polyols production in DMC materials. First, the $\mathrm{Zn}$ active sites are as isolated as possible and present coordination spheres with ligands other than N. O atoms as linkers seem especially suited to adjust the properties of $\mathrm{Zn}$ from an electronic point of view. However, $\mathrm{Zn}-\mathrm{O}$ structures are labile and thus 
are prone to be modified, added to the polymer as ligands, and impose a large dynamicity to the active center, which opens more active sites along the reaction of centers with more $\mathrm{O}$ ligands. However, these can be more likely to interchange among the ligands and the reactant and can lead to some degree of multiple head growth. It has been proposed in Ref. [12] that a protic acid can alleviate the formation of highweight tails. By revising our data, we can infer the reason behind the positive role of carboxylate acids. Acids can protonate $C A$, which breaks the short $Z n-Z n$ contacts that we have described as detrimental because of covalent interactions. Moreover, as the CAs are labile they can be replaced by the carboxylic conjugated bases. These linkers are also labile ${ }^{[25]}$ but they are less susceptible to be transferred to the growing polyol because of the dihapto nature of these linkers. In addition, protons can interact with the $\mathrm{OH}$ groups of the polyol to make the growing polymer more "linear" and reduce the probability of chain entangling through noncovalent mechanisms.

\section{Conclusions}

We have shown the most salient atomic features of double metal cyanide catalysts in the polymerization of epoxides by means of theoretical simulations based on DFT. Even if the complexity of the system is far beyond the abilities of the state-of-the-art modeling techniques, the investigation of three key steps in the initialization and the first propagation steps can shed light on the process. Indeed, although the degree of amorphization is crucial to long-term operation, the local geometrical patterns are reasonably well reproduced in the simulations of the optimized crystals. Therefore, the most exposed surfaces contain two types of active centers: one with two $\mathrm{Zn}$ atoms at a short distance and a second for which the $\mathrm{Zn}-\mathrm{Zn}$ contacts are in the range of approximately $7 \AA$. The activity of isolated atoms with a coordination of $\mathrm{Zn}$ depends on the coordination sphere. $\mathrm{N}$-containing ligands establish a crystal field that allows interaction with incoming epoxides, but O-containing ligands improve activity. Therefore, $\mathrm{Zn}$ atoms surrounded by at least two O-containing groups are needed as active sites. However, this configuration imposes a large degree of lability on the structure and can cause the formation of chains with multiple reactive elongation sites because of the chemical interference between the centers. Physical phenomena such as intercrossing cannot be discarded based on catalyst topology either, although this can be addressed by using surfaces with more spaced catalytic centers. Finally, the addition of protic acids can lead to a lower amount of high-weight tails as they can cleave the redundant or new $\mathrm{OH}$ groups among $\mathrm{Zn}$ atoms and smooth the lability of the catalysts. Simultaneously, proton addition can lead to less convoluted polymers that are less susceptible to noncovalent interactions.

\section{Experimental Section}

The structural and catalytic properties of DMC catalysts were investigated by DFT using the VASP code. ${ }^{[28]}$ The Perdew-Burke-Ernzerhof (PBE) functional was chosen. ${ }^{[29]}$ Inner electrons were replaced by projector augmented wave (PAW) frozen cores, ${ }^{[28 c, 30]}$ and valence monoelectronic states were expanded in plane waves with kinetic cut-off energy of $450 \mathrm{eV}$. The $k$-point sampling was set to be denser than $0.66 \AA^{-1}$ for bulk calculations. ${ }^{[3]]}$ The crystal structure of the DMC was obtained from Ref. [23]. For all the CA-modified systems, all the $\mathrm{Cl}$ atoms in the structure were replaced by the $\mathrm{CA}(\mathrm{OH}, \mathrm{Cl}, \mathrm{OEt}, \mathrm{OtBu})$ and the bulk was reoptimized with the same set up. The two surfaces with low Miller indices, (001) and (100), were also investigated by a k-point density of $0.40 \AA^{-1}$. These surfaces were modeled as periodically repeated slabs that contain two layers of $\mathrm{Co}(\mathrm{CN})_{6}{ }^{3-}$ and four layers of $\mathrm{Zn}^{2+}$ that were interleaved by a $10 \AA$ vacuum. The dipole moments that arise as a result of the asymmetry of the lattice were then removed following standard dipole corrections. To keep the stoichiometry of the material if the slabs were cut, capping $\mathrm{OH}$ groups were added. The equations for the surface energy were modified to represent the energies from the equations: $\mathrm{C}_{12} \mathrm{~N}_{12} \mathrm{Zn}_{4} \mathrm{CO}_{2}(\mathrm{OH})_{2}+\mathrm{H}_{2} \mathrm{O} \rightarrow$ $\mathrm{C}_{12} \mathrm{~N}_{12} \mathrm{Zn}_{4} \mathrm{CO}_{2}(\mathrm{OH})_{2}-\mathrm{OH}(001)+1 / 2 \mathrm{H}_{2} ; \quad 2 \mathrm{C}_{12} \mathrm{~N}_{12} \mathrm{Zn}_{4} \mathrm{Co}_{2}(\mathrm{OH})_{2}+2 \mathrm{H}_{2} \mathrm{O} \rightarrow$ $2 \mathrm{C}_{12} \mathrm{~N}_{12} \mathrm{Zn}_{4} \mathrm{Co}_{2}(\mathrm{OH})_{2}-4 \mathrm{OH}(100)+2 \mathrm{H}_{2}$, which is related to water and hydrogen reservoirs. Large and variable $\mathrm{OH}$ contents have been found for several of these materials as demonstrated by IR spectroscopy. ${ }^{[10,18,32]}$ The surface energies were then obtained with a one side relaxation scheme as follows $\gamma=$ ( $\left.E_{\text {unrelax-slab }}-N_{\text {bulk }} E_{\text {bulk }}-N_{\mathrm{OH}} E_{\mathrm{H}_{2} \mathrm{O}}+0.5 N_{\mathrm{OH}} E_{\mathrm{H}_{2}}\right) / 2 A+\left(E_{\text {relax-slab }}-E_{\text {unrelax-slab }}\right) /$ $A$. In which $E_{\text {unrelax-slab }}$ and $E_{\text {relax-slab }}$ are the unrelaxed and relaxed slab energies, $E_{\text {bulk }}$ is that of the bulk, $N_{\text {bulk }}$ is the number of formula units, $N_{\mathrm{OH}}$ is the number of hydroxyl fragments, $E_{\mathrm{H}_{2} \mathrm{O}} E_{\mathrm{H}_{2}}$ are the reference gas-phase energies, and $A$ is the surface area. The dipole moments that arise because of the asymmetry of the lattice were then removed following standard dipole corrections. A $(2 \times 1)$ supercell was employed to describe the reactivity on the $(001)$ facet, and a $(1 \times 1)$ supercell was used for the larger $(100)$ facet. Although in principle the unit cells might seem small, there are two technical issues that justify our selection: 1 ) the $\mathrm{Zn}$ atoms are rather separated in an isolated site fashion and the unit cells are rather large because of the presence of spacers, 2) the polymerization takes place in a very dense environment as the pressure of the epoxide is approximately 4 bar and the polymer grows to cover the catalyst surface completely, thus the coverage under reaction conditions is one chain per active site. The location of the transition states was identified by employing the climbing image version of the nudged elastic band method (Cl-NEB) ${ }^{[33]}$ with an optimization threshold of $0.05 \mathrm{eV}$. The saddle nature of the transition state structures was obtained by the diagonalization of the numerical Hessian generated by $0.02 \AA$ steps.

\section{Acknowledgements}

We would like to thank REPSOL for supporting this research.

Keywords: cobalt - density functional theory $\cdot$ double metal cyanides $\cdot$ polymerization $\cdot$ reaction mechanisms $\cdot$ zinc

[1] S. Seneker, N. Barksby, Am. Chem. Coun. 1996, 305.

[2] a) H. R. Johnston, US3278459, 1966; b) M. Jack, US3404109, 1968; c) J. Milgrom, US404109, 1968; d) R. J. Herold, R. A. Livigni, Polym. Prepr. Am. Chem. Soc. Div. Polym. Chem. 1972, 13, 545; e) A. Livigni, R. J. Herold, O. C. Elmer, S. L. Aggarwal in Polyethers, Vol. 6, American Chemical Society, Washington, DC, 1975, pp. 20-37; f) R. J. Herold, Macromol. Synth 1974, 5, 9.

[3] D. F. Mullica, W. O. Milligan, G. W. Beall, W. L. Reeves, Acta Crystallogr. Sect. B 1978, 34, 3558-3561.

[4] B. Le-Khac, EP 19970919340, 2001. 
[5] B. Le-Khac, US5470813, 1995.

[6] X.-H. Zhang, Z.-J. Hua, S. Chen, F. Liu, X.-K. Sun, G.-R. Qi, Appl. Catal. A 2007, 325, $91-98$

[7] a) R. J. Belner, US3427334, 1969; b) M. Jack, US3427256, 1969.

[8] I. Kim, K. Anas, S. Lee, C.-S. Ha, D.-W. Park, Catal. Today 2008, 131, 541 547.

[9] P. T. Bowman, H. R. Hinney, B. Le-Khac, EP 0743093 B1, 2001.

[10] S. Chen, P. Zhang, L. Chen, Prog. Org. Coat. 2004, 50, 269-272.

[11] S. Chen, L. Chen, Colloid Polym. Sci. 2004, 282, 1033-1038.

[12] M. K. Faraj, B. Le-Khac, W. Wang, US6063897, 2000

[13] K. Lee, E. D. Murray, L. Kong, B. I. Lundqvist, D. C. Langreth, Phys. Rev. B 2010, 82, 081101.

[14] I. Kim, J. T. Ahn, C. S. Ha, C. S. Yang, I. Park, Polymer 2003, 44, $3417-$ 3428.

[15] a) X. Liu, M. Kang, X. Wang, Hecheng Xiangjiao Gongye 2002, 14, 247; b) X. Liu, M. Kang, X. Wang, Hecheng Xiangjiao Gongye 2002, 24, 147.

[16] H. Yin, Z. R. Chen, Chin. J. Chem. Eng. 2002, 10, 218-222.

[17] Y. Dienes, W. Leitner, M. G. J. Mueller, W. K. Offermans, T. Reier, A. Reinholdt, T. E. Weirich, T. E. Mueller, Green Chem. 2012, 14, 1168- 1177.

[18] I. K. Lee, J. Y. Ha, C. Cao, D.-W. Park, C.-S. Ha, I. Kim, Catal. Today 2009, 148, 389-397.

[19] D. Du, M. Cao, X. He, Y. Liu, C. Hu, Langmuir 2009, 25, 7057-7062.

[20] S. S. Kaye, J. R. Long, J. Am. Chem. Soc. 2005, 127, 6506-6507.

[21] N. López, N. Almora-Barrios, G. Carchini, P. Błonski, L. Bellarosa, R. García-Muelas, G. Novell-Leruth, M. García-Mota, Catal. Sci. Technol. 2012, 2, 2405-2417.
[22] D. Di Tommaso, R. I. Ainsworth, E. Tang, N. H. de Leeuw, J. Mater. Chem. B 2013, 1, 5054-5066.

[23] J. C. Wojdeł, S. T. Bromley, F. Illas, J. C. Jansen, J. Mol. Model. 2007, 13, $751-756$.

[24] P. J. Flory, Principles of Polymer Chemistry, Cornell University Press, New York, 1953.

[25] L. Bellarosa, J. M. Castillo, T. Vlugt, S. Calero, N. Lopez, Chem. Eur. J. 2012, 18, 12260-12266.

[26] J. J. Lear, J. F. Pazos, O. D. Sloan, 6083420, 2000.

[27] M. Doi, S. F. Edwards, J. Chem. Soc. Faraday Trans. 1978, 74, 1789-1801.

[28] a) G. Kresse, J. Furthmuller, Phys. Rev. B 1996, 54, 11169-11186; b) G. Kresse, J. Furthmuller, Comput. Mater. Sci. 1996, 6, 15-50; c) G. Kresse, D. Joubert, Phys. Rev. B 1999, 59, 1758-1775.

[29] J. P. Perdew, K. Burke, M. Ernzerhof, Phys. Rev. Lett. 1996, 77, 3865 3868.

[30] T. Bucko, J. Hafner, S. Lebègue, J. G. Ángyán, J. Phys. Chem. A 2010, 114 , $11814-11824$.

[31] M. Methfessel, A. T. Paxton, Phys. Rev. B 1989, 40, 3616-3621.

[32] B. Le-Khac, EP 0700949 B1, 1999.

[33] G. Henkelman, B. P. Uberuaga, H. Jónsson, J. Chem. Phys. 2000, 113, 9901-9904. 